\title{
Line Balancing for Improving Apparel Production by Operator Skill Matrix
}

\author{
Md. Mazharul Islam ${ }^{1, ~ *, ~ M d . ~ T a n j i m ~ H o s s a i n ~}{ }^{1}$, Mohammad Abdul Jalil ${ }^{2}$, Elias Khalil ${ }^{3}$ \\ ${ }^{1}$ Department of Textile Engineering, Northern University of Bangladesh, Dhaka, Bangladesh \\ ${ }^{2}$ Department of Textile Engineering, Mawlana Bhashani Science and Technology University, Tangail, Bangladesh \\ ${ }^{3}$ Department of Textile Engineering, World University of Bangladesh, Dhaka, Bangladesh \\ Email address: \\ mazh999@gmail.com (Md. M. Islam),tanjimseu@yahoo.com (Md. T. Hossain), jalil.mbstu@gmail.com (M. A. Jalil), \\ eliaskhali152@gmail.com (E. Khalil)
}

\section{To cite this article:}

Md. Mazharul Islam, Md. Tanjim Hossain, Mohammad Abdul Jalil, Elias Khalil. Line Balancing for Improving Apparel Production by Operator Skill Matrix. International Journal of Science, Technology and Society. Vol. 3, No. 4, 2015, pp. 101-106.

doi: $10.11648 /$ j.ijsts.20150304.11

\begin{abstract}
In this modern world, fashion \& styles are changing frequently. The emergence of fast changes in fashion has given rise to shorten production cycle time in the garment industry. To meet the dynamic customer demands of momentous quantities in shorten lead time, assembly line production systems are used, where the garment components are assembled into a finished garment through a sub-assembly process. So in the era of product customization, the optimal usage of resources especially the available facilities \& operators who are adding the value of product is important. Therefore the assembly line has to be planned in much more flexible way. This paper deals with the maximum utilization of manpower in labor intensive assembly lines. The objective is to accurately delegate workers to the various operations required to complete the product based on their skill \& experience so as to achieve the highest level of productivity and delivery as per planned target. The experimental result showed meaningful improvement in productivity as compared to the existing system.
\end{abstract}

Keywords: Line Balancing, Skill Matrix, Assembly Line, Apparel Production

\section{Introduction}

As a supply chain of textile industry, garment industry is one of the major industries of the world. The production process of garments is separated into four main phases: designing/ clothing pattern generation, fabric spreading \& cutting, sewing and ironing \& packing. The most critical phase is sewing phase [1]. As the sewing is the heart of apparel industry, we have to design the sewing line properly so as to achieve the best output at maximum efficiency. Apparel is a mass production system. Assembly line production systems are developed to meet the requirements of mankind, which continue to grow day by day [2]. The demand for greater product variability and shorter life cycles has caused traditional production methods to be replaced with assembly lines [3]. Assembly line is an industrial arrangement of machines, equipments and workers for continuous flow of work pieces in mass production operation. Manufacturing a product in an assembly line requires partitioning the total amount of work into a set of elementary operations called tasks [4]. Tasks are assigned to operators depending on constrains of different labor skill levels. Finally, several workstations in sequence are formed as a sewing line [5]. The sewing process includes a set of work stations, at each of which a specific task is carried out in a restricted sequence, with hundreds of employees and thousands of bundles of sub-assemblies producing different style simultaneously [6]. Therefore this process is of critical importance and needs to be planned more carefully [7]. As a consequence, good line balancing with small stocks in the sewing line has to be drawn up to increase the efficiency and quality [7-9]. So the aim of assembly line balancing in sewing line is to assign tasks to the workstations, so that the machines of the workstation can perform the assigned task with a balanced loading with different labor skill levels [5].

\section{Literature Review}

A line is defined as a group of operators under the control of one production supervisor [10]. Balancing is the technique of maintaining the same level of inventory at each and every 
operation at any point of time to meet the production target and to produce garments of acceptable quality [10]. Assembly line balancing (ALB) is a managerial technique and can be applied to balance production flow lines [11-12]. Line balancing is the distribution of work on the line in such a way that everyone gets the same amount of work in terms of time [13]. In practice, a perfect balance could not be achieved but we can improve the situation by using proper technique.

The assembly line balancing problem was first introduced by Bryton in his graduate thesis. In his study, he accepted the amount of workstations as constant, the workstation times as equal for all stations and work tasks as moving among the workstations [14]. The first article was published in 1955 by Salveson [15]. He developed a 0-1 integer programming model to solve the problem. This heuristic method was developed by Helgeson and Birnie of the General Electric Company in 1961 COMSOAL (Computer Method of Sequencing Operations for Assembly Lines) was first used by Arcus in 1966 as a solution approach to the assembly line balancing problem [16,17]. Bartholdi (1993) was the first to address the Two-sided Assembly Line Balancing Problem with the objective of minimizing the number of stations by applying a simple assignment rule. Liu \& Chen (2002) presented a Genetic Algorithm approach for assembly planning involving various objectives, such as minimizing cycle time, maximizing workload smoothness, minimizing the frequency of tool change, minimizing the number of tools and machines used and minimizing the complexity of assembly sequences [18-19]. Helgeson ve Birnie (1961) developed the "Ranked Positional Weight Technique" in which operation having the largest ranged weight is assigned to the first workstation, and other operations are assigned to workstations in accordance with their ranked positional weight value [16]. Abdolmajid Yolmeh et al. (2012) proposed a hybrid genetic algorithm to solve the assembly line balancing problem [20].

Operator's Skill Inventory is the database which maintains the record of each operator, who can do what type of operation and at what rating. It is very important to keep this database updated as over the time, operator acquire skills for most of the new operations as well as improve performance in existing operations [21].With the use of skill matrix an engineer's or line supervisors needs very list time to find out and select most efficient operator for a particular operation from the pull of operation. It helps the line supervisor for balancing the line with particular skilled operators according to the work content [21].

There are so many researches going on in the field of assembly line balancing. Various methods are used for balancing sewing lines which are discussed in the above section. We use operator skill matrix for better allocation of operator throughout the sewing line to get maximum output.

\section{Materials and Methodology}

In this experiment, $100 \%$ cotton Jacket was considered. Total 30 sewing machines were used where number of plain, over lock and flat lock machines was 13, 11, and 6 respectively.

In order to balance a production line in sewing floor a line was chosen $\&$ necessary data was accumulated from the line. First the garment was analyzed and operational bulletin or breakdown was created with process sequence, operational description \& machine requirements (Table 1). Then workers were placed to different work stations based on operation \& machine types and a standard minute for each job was given to the operators (allocated SMV) (Table 1). After one day we calculate the output \& found that we didn't get the desired output. To found out the problem, we calculated individual workers performed SMV by work study. After that workers individual efficiency \& output at individual efficiency was calculated and then saw that efficiencies varies dramatically due to unplanned layout (Table 1). So to balance the line we have to rearrange the operators within the line. To do this, first machine-wise breakdown (Table 2 to Table 7) was done and workers are reallocated based on two assumptions: a) operators are only be allocated depending on the machine type that he/she can operate; b) allocation is also depend on operation type that he/she can perform. After fulfilling above two conditions we rearranged operators based on SMV. Higher the SMV, higher should be the efficiency \% as we know where the workload is high, we need higher efficient worker. After total rearrangement, we again calculated the output (Table 8). We observed that productivity is increased but not up to the theoretical productivity. In some process, huge bottleneck was appeared. So to improve productivity we introduced another worker to the bottleneck operation by dividing the workload between two workers (Table 9). Finally we got satisfactory productivity.

\section{Experimental Data}

Operational Bulletin of Jacket before and after arrangement, Breakdown of different machines before and after rearrangement along with comparison of productions are shown below in different tables.

Table 1. Operational Bulletin of Jacket before arrangement.

\begin{tabular}{|c|c|c|c|c|c|c|c|c|}
\hline SL & Process Name & Name & M/c Type & $\begin{array}{l}\text { Performed } \\
\text { SMV }\end{array}$ & $\begin{array}{l}\text { Allocated } \\
\text { SMV }\end{array}$ & $\begin{array}{l}\text { Output@ } \\
100 \% \text { eff }\end{array}$ & Efficiency \% & $\begin{array}{l}\text { Output@ } \\
\text { performed } \\
\text { efficiency \% }\end{array}$ \\
\hline 1 & Back part panel join & Jarna & $\mathrm{O} / \mathrm{L}$ & 0.64 & 0.62 & 96.77 & 96 & 93.26 \\
\hline 2 & Back panel ts tc & Naher & $\mathrm{F} / \mathrm{L}$ & 0.35 & 0.3 & 200.00 & 87 & 173.08 \\
\hline 3 & Left and right panel join & Hasina & $\mathrm{O} / \mathrm{L}$ & 1.72 & 0.62 & 96.77 & 36 & 34.95 \\
\hline 4 & Left and right panel join & Eima & $\mathrm{O} / \mathrm{L}$ & 1.28 & 0.62 & 96.77 & 48 & 46.88 \\
\hline 5 & Left and right panel ts and tc & Mena & $\mathrm{F} / \mathrm{L}$ & 0.62 & 0.55 & 109.09 & 89 & 97.30 \\
\hline
\end{tabular}




\begin{tabular}{|c|c|c|c|c|c|c|c|c|}
\hline SL & Process Name & Name & M/c Type & $\begin{array}{l}\text { Performed } \\
\text { SMV }\end{array}$ & $\begin{array}{l}\text { Allocated } \\
\text { SMV }\end{array}$ & $\begin{array}{l}\text { Output@ } \\
100 \% \text { eff }\end{array}$ & Efficiency \% & $\begin{array}{l}\text { Output @ } \\
\text { performed } \\
\text { efficiency } \%\end{array}$ \\
\hline 6 & Front part panel join \& tc & Ronju & $\mathrm{O} / \mathrm{L}$ & 0.78 & 0.5 & 120.00 & 64 & 76.60 \\
\hline 7 & Front part panel ts \& tc & Monzilla & $\mathrm{L} / \mathrm{S}$ & 0.53 & 0.3 & 200.00 & 57 & 113.92 \\
\hline 8 & Left \& right panel join & Amina & $\mathrm{O} / \mathrm{L}$ & 0.82 & 0.62 & 96.77 & 76 & 73.47 \\
\hline 9 & Left and right panel join & Sahina & $\mathrm{O} / \mathrm{L}$ & 0.79 & 0.62 & 96.77 & 78 & 75.95 \\
\hline 10 & Left and right panel ts \& tc & Nurbanu & $\mathrm{F} / \mathrm{L}$ & 0.53 & 0.55 & 109.09 & 103 & 112.50 \\
\hline 11 & Shoulder panel join & Lima & $\mathrm{O} / \mathrm{L}$ & 0.94 & 0.43 & 139.53 & 46 & 63.60 \\
\hline 12 & Shoulder panel ts & Alima & $\mathrm{F} / \mathrm{L}$ & 0.88 & 0.42 & 142.86 & 48 & 68.18 \\
\hline 13 & Shoulder join & Rojina & $\mathrm{O} / \mathrm{L}$ & 0.92 & 0.59 & 101.69 & 64 & 65.45 \\
\hline 14 & Sleeve panel make & Morina & $\mathrm{O} / \mathrm{L}$ & 0.64 & 0.45 & 133.33 & 70 & 93.26 \\
\hline 15 & Sleeve panel top stitch \& tc & Sokina & $\mathrm{F} / \mathrm{L}$ & 0.47 & 0.49 & 122.45 & 104 & 126.76 \\
\hline 16 & Sleeve hem & Momina & $F / L$ & 0.42 & 0.3 & 200.00 & 71 & 141.73 \\
\hline 17 & Collar make & Pervin & $\mathrm{L} / \mathrm{S}$ & 0.79 & 0.63 & 95.24 & 80 & 75.95 \\
\hline 18 & Collar twill tape attach & Rebeka & $\mathrm{L} / \mathrm{S}$ & 0.26 & 0.22 & 272.73 & 85 & 230.77 \\
\hline 19 & Collar serving & Roksana & $\mathrm{O} / \mathrm{L}$ & 0.43 & 0.39 & 153.85 & 90 & 138.46 \\
\hline 20 & Zipper twill tape make & Sabina & $\mathrm{L} / \mathrm{S}$ & 0.88 & 0.42 & 142.86 & 48 & 68.44 \\
\hline 21 & Zipper twill tape attach & Rojina & $\mathrm{L} / \mathrm{S}$ & 0.87 & 0.5 & 120.00 & 57 & 68.97 \\
\hline 22 & Zipper holding tuck & Bobita & $\mathrm{L} / \mathrm{S}$ & 0.66 & 0.3 & 200.00 & 45 & 90.91 \\
\hline 23 & Zipper patch attach at bottom & Shahanara & $\mathrm{L} / \mathrm{S}$ & 0.45 & 0.57 & 105.26 & 127 & 133.33 \\
\hline 24 & Collar join & Sabina & $\mathrm{L} / \mathrm{S}$ & 1.77 & 0.78 & 76.92 & 44 & 33.90 \\
\hline 25 & Collar join & Shahila & $\mathrm{L} / \mathrm{S}$ & 1.99 & 0.78 & 76.92 & 39 & 30.15 \\
\hline 26 & Zipper tuck with body & Naher & $\mathrm{L} / \mathrm{S}$ & 0.36 & 0.42 & 142.86 & 118 & 168.22 \\
\hline 27 & Zipper join with left side & Halima & $\mathrm{L} / \mathrm{S}$ & 0.58 & 0.5 & 120.00 & 86 & 103.45 \\
\hline 28 & Zipper join right side & Orchona & $\mathrm{L} / \mathrm{S}$ & 0.76 & 0.69 & 86.96 & 90 & 78.60 \\
\hline 29 & Zipper facing join & Beauty & $\mathrm{L} / \mathrm{S}$ & 0.66 & 0.5 & 120.00 & 76 & 90.91 \\
\hline 30 & Zipper facing o/l & Sharmin & $\mathrm{O} / \mathrm{L}$ & 0.36 & 0.3 & 200.00 & & 165.14 \\
\hline & & & & \multicolumn{2}{|c|}{ Max Theoretical output } & 76.92 & practical output & $30 \mathrm{pcs}$ \\
\hline
\end{tabular}

Table 2. Breakdown of Plain machines before rearrangement.

\begin{tabular}{|c|c|c|c|c|c|}
\hline SL & Process Name & Name & M/c Type & Performed SMV & Efficiency \\
\hline 7 & Front part panel ts \& tc & Monzilla & $\mathrm{L} / \mathrm{S}$ & 0.53 & $57 \%$ \\
\hline 17 & Collar make & Pervin & $\mathrm{L} / \mathrm{S}$ & 0.79 & $80 \%$ \\
\hline 18 & Collar twill tape attach & Rebeka & $\mathrm{L} / \mathrm{S}$ & 0.26 & $85 \%$ \\
\hline 20 & Zipper twill tape make & Sabina & $\mathrm{L} / \mathrm{S}$ & 0.88 & $48 \%$ \\
\hline 21 & Zipper twill ta[e attach & Rojina & $\mathrm{L} / \mathrm{S}$ & 0.87 & $57 \%$ \\
\hline 22 & Zipper holding tuck & Bobita & $\mathrm{L} / \mathrm{S}$ & 0.66 & $45 \%$ \\
\hline 23 & Zipper patch attach at bottom & Shahanara & $\mathrm{L} / \mathrm{S}$ & 0.45 & $127 \%$ \\
\hline 24 & Collar join & Sabina & $\mathrm{L} / \mathrm{S}$ & 1.77 & $44 \%$ \\
\hline 25 & Collar join & Shahila & $\mathrm{L} / \mathrm{S}$ & 1.99 & $39 \%$ \\
\hline 26 & Zipper tuck with body & Naher & $\mathrm{L} / \mathrm{S}$ & 0.36 & $118 \%$ \\
\hline 27 & Zipper join with left side & Halima & $\mathrm{L} / \mathrm{S}$ & 0.58 & $86 \%$ \\
\hline 28 & Zipper join right side & Orchona & $\mathrm{L} / \mathrm{S}$ & 0.76 & $90 \%$ \\
\hline 29 & Zipper facing join & Beauty & $\mathrm{L} / \mathrm{S}$ & 0.66 & $76 \%$ \\
\hline
\end{tabular}

Table 3. Breakdown of Plain machines after rearrangement.

\begin{tabular}{llllll}
\hline SL & Process Name & Name & M/c Type & Performed SMV & Efficiency \\
\hline 18 & Collar twill tape attach & Shahila & L/S & 0.26 & $39 \%$ \\
26 & Zipper tuck with body & Sabina & L/S & 0.36 & $44 \%$ \\
23 & Zipper patch attach at bottom & Bobita & L/S & 0.45 & $45 \%$ \\
7 & Front part panel ts \& tc & Sabina & L/S & 0.53 & $48 \%$ \\
27 & Zipper join with left side & Monzilla & L/S & 0.58 & $57 \%$ \\
22 & Zipper holding tuck & Rojina & L/S & 0.66 & $57 \%$ \\
29 & Zipper facing join & Beauty & L/S & 0.66 & $76 \%$ \\
28 & Zipper join right side & Pervin & L/S & 0.76 & $80 \%$ \\
17 & Collar make & Rebeka & L/S & 0.79 & $85 \%$ \\
21 & Zipper twill tape attach & Halima & L/S & 0.87 & $86 \%$ \\
20 & Zipper twill tape make & Orchona & L/S & 0.88 & 1.77 \\
24 & Collar join & Naher & L/S & 1.99 & $118 \%$ \\
25 & Collar join & Shahanara & L/S & & $127 \%$ \\
\hline
\end{tabular}


Table 4. Breakdown of Overlock machines before rearrangement.

\begin{tabular}{|c|c|c|c|c|c|}
\hline SN & Process Name & Name & M/c Type & Performed SMV & Efficiency \\
\hline 1 & Back part panel join & Jarna & $\mathrm{O} / \mathrm{L}$ & 0.64 & $96 \%$ \\
\hline 3 & Left and right panel join & Hasina & $\mathrm{O} / \mathrm{L}$ & 1.72 & $36 \%$ \\
\hline 4 & Left and right panel join & Eima & $\mathrm{O} / \mathrm{L}$ & 1.28 & $48 \%$ \\
\hline 6 & Front part panel join \& tc & Ronju & $\mathrm{O} / \mathrm{L}$ & 0.78 & $64 \%$ \\
\hline 8 & Left \& right panel join & Amina & $\mathrm{O} / \mathrm{L}$ & 0.82 & $76 \%$ \\
\hline 9 & Left and right panel join & Sahina & $\mathrm{O} / \mathrm{L}$ & 0.79 & $78 \%$ \\
\hline 11 & Shoulder panel join & Lima & $\mathrm{O} / \mathrm{L}$ & 0.94 & $46 \%$ \\
\hline 13 & Shoulder join & Rojina & $\mathrm{O} / \mathrm{L}$ & 0.92 & $64 \%$ \\
\hline 14 & Sleeve panel make & Morina & $\mathrm{O} / \mathrm{L}$ & 0.64 & $70 \%$ \\
\hline 19 & Collar serving & Roksana & $\mathrm{O} / \mathrm{L}$ & 0.43 & $90 \%$ \\
\hline 30 & Zipper facing o/l & Sharmin & $\mathrm{O} / \mathrm{L}$ & 0.36 & $83 \%$ \\
\hline
\end{tabular}

Table 5. Breakdown of Overlock machines after rearrangement.

\begin{tabular}{llllll}
\hline SN & Process Name & Name & M/c Type & Performed SMV & Efficiency \\
\hline 30 & Zipper facing o/l & Hasina & $\mathrm{O} / \mathrm{L}$ & 0.36 & $36 \%$ \\
19 & Collar serving & Lima & $\mathrm{O} / \mathrm{L}$ & 0.43 & $46 \%$ \\
14 & Sleeve panel make & Eima & $\mathrm{O} / \mathrm{L}$ & 0.64 & $48 \%$ \\
1 & Back part panel join & Ronju & $\mathrm{O} / \mathrm{L}$ & 0.64 & $64 \%$ \\
6 & Front part panel join \& tc & Rojina & $\mathrm{O} / \mathrm{L}$ & 0.78 & $64 \%$ \\
9 & Left and right panel join & Morina & $\mathrm{O} / \mathrm{L}$ & 0.79 & $70 \%$ \\
8 & Left \& right panel join & Amina & $\mathrm{O} / \mathrm{L}$ & 0.82 & $76 \%$ \\
13 & Shoulder join & Sahina & $\mathrm{O} / \mathrm{L}$ & 0.92 & $78 \%$ \\
11 & Shoulder panel join & Sharmin & $\mathrm{O} / \mathrm{L}$ & 0.94 & $83 \%$ \\
4 & Left and right panel join & Roksana & $\mathrm{O} / \mathrm{L}$ & 1.28 & $90 \%$ \\
3 & Left and right panel join & Jarna & $\mathrm{O} / \mathrm{L}$ & 1.72 & $96 \%$ \\
\hline
\end{tabular}

Table 6. Breakdown of Flat lock machines before rearrangement.

\begin{tabular}{llllll}
\hline SN & Process Name & Name & M/c Type & Performed SMV & Efficiency \\
\hline 2 & Back panel ts tc & Naher & F/L & 0.35 & $87 \%$ \\
5 & Left and right panel ts and tc & Mena & F/L & 0.62 & $89 \%$ \\
10 & Left and right panel ts \& tc & Nurbanu & F/L & 0.53 & $103 \%$ \\
12 & Shoulder panel ts & Alima & F/L & 0.88 & $48 \%$ \\
15 & Sleeve panel top stitch \& tc & Sokina & F/L & 0.47 & $104 \%$ \\
16 & Sleeve hem & Momina & F/L & 0.42 & $71 \%$ \\
\hline
\end{tabular}

Table 7. Breakdown of Flat lock machines after rearrangement.

\begin{tabular}{llllll}
\hline SN & Process Name & Name & m/c & Performed SMV & Efficiency \\
\hline 2 & Back panel ts tc & Alima & F/L & 0.35 & $48 \%$ \\
16 & Sleeve hem & Momina & F/L & 0.42 & $71 \%$ \\
15 & Sleeve panel top stitch \& tc & Naher & F/L & 0.47 & $87 \%$ \\
10 & Left and right panel ts \& tc & Mena & F/L & 0.53 & $89 \%$ \\
5 & Left and right panel ts and tc & Nurbanu & F/L & 0.62 & $103 \%$ \\
12 & Shoulder panel ts & Sokina & F/L & 0.88 & $104 \%$ \\
\hline
\end{tabular}

Table 8. Operational Bulletin of Jacket after rearrangement.

\begin{tabular}{lllllll}
\hline SN & Process Name & Name & M/c Type & Performed SMV & Efficiency \% & Output @ performed efficiency \\
\hline 1 & Back part panel join & Ronju & O/L & 0.64 & 48 & 46.45 \\
2 & Back panel ts tc & Alima & F/L & 0.35 & 48 & 96.00 \\
3 & Left and right panel join & Jarna & O/L & 1.72 & 96 & 92.90 \\
4 & Left and right panel join & Roksana & O/L & 1.28 & 90 & 87.10 \\
5 & Left and right panel ts and tc & Nurbanu & F/L & 0.62 & 71 & 77.45 \\
6 & Front part panel join \& tc & Rojina & O/L & 0.78 & 64 & 76.80 \\
7 & Front part panel ts \& tc & Sabina & L/S & 0.53 & 44 & 88.00 \\
8 & Left \& right panel join & Amina & O/L & 0.82 & 76 & 73.55 \\
9 & Left and right panel join & Morina & O/L & 0.79 & 70 & 67.74 \\
10 & Left and right panel ts \& tc & Mena & F/L & 0.53 & 87 & 94.91 \\
11 & Shoulder panel join & Sharmin & O/L & 0.94 & 83 & 115.81 \\
\hline
\end{tabular}




\begin{tabular}{lllllll}
\hline SN & Process Name & Name & M/c Type & Performed SMV & Efficiency \% & Output a performed efficiency \\
\hline 12 & Shoulder panel ts & Sokina & F/L & 0.88 & 89 & 127.14 \\
13 & Shoulder join & Sahina & O/L & 0.92 & 78 & 79.32 \\
14 & Sleeve panel make & Eima & O/L & 0.64 & 64 & 85.33 \\
15 & Sleeve panel top stitch \& tc & Naher & F/L & 0.47 & 103 & 126.12 \\
16 & Sleeve hem & Momina & F/L & 0.42 & 104 & 208.00 \\
17 & Collar make & Rebeka & L/S & 0.79 & 86 & 81.90 \\
18 & Collar twill tape attach & Shahila & L/S & 0.26 & 39 & 106.36 \\
19 & Collar serving & Lima & O/L & 0.43 & 46 & 70.77 \\
20 & Zipper twill tape make & Orchona & L/S & 0.88 & 48 & 68.57 \\
21 & Zipper twill tape attach & Halima & L/S & 0.87 & 57 & 68.40 \\
22 & Zipper holding tuck & Rojina & L/S & 0.66 & 45 & 90.00 \\
23 & Zipper patch attach at bottom & Bobita & L/S & 0.45 & 85 & 89.47 \\
24 & Collar join & Naher & L/S & 1.77 & 118 & 90.77 \\
25 & Collar join & Shahanara & L/S & 1.99 & 127 & 97.69 \\
26 & Zipper tuck with body & Sabina & L/S & 0.36 & 57 & 81.43 \\
27 & Zipper join with left side & Monzilla & L/S & 0.58 & 76 & 91.20 \\
28 & Zipper join right side & Pervin & L/S & 0.76 & 90 & 78.26 \\
29 & Zipper facing join & Beauty & L/S & 0.66 & 80 & 96.00 \\
30 & Zipper facing o/l & Hasina & O/L & 0.36 & 36 & 72.00 \\
\hline
\end{tabular}

Practical output after rearrangement

Table 9. Final Practical output after sharing of work.

\begin{tabular}{|c|c|c|c|c|c|c|}
\hline SN & Process Name & Name & M/c Type & Performed SMV & Efficiency \% & Output @ performed efficiency \\
\hline 1 & Back part panel join & Raju & $\mathrm{O} / \mathrm{L}$ & 0.64 & 48 & 46.45 \\
\hline 1 & Back part panel join & Ronju & $\mathrm{O} / \mathrm{L}$ & 0.64 & 48 & 46.45 \\
\hline 2 & Back panel ts tc & Alima & $\mathrm{F} / \mathrm{L}$ & 0.35 & 48 & 96.00 \\
\hline 3 & Left and right panel join & Jarna & $\mathrm{O} / \mathrm{L}$ & 1.72 & 96 & 92.90 \\
\hline 4 & Left and right panel join & Roksana & $\mathrm{O} / \mathrm{L}$ & 1.28 & 90 & 87.10 \\
\hline 5 & Left and right panel ts and tc & Nurbanu & $\mathrm{F} / \mathrm{L}$ & 0.62 & 71 & 77.45 \\
\hline 6 & Front part panel join \& tc & Rojina & $\mathrm{O} / \mathrm{L}$ & 0.78 & 64 & 76.80 \\
\hline 7 & Front part panel ts \& tc & Sabina & $\mathrm{L} / \mathrm{S}$ & 0.53 & 44 & 88.00 \\
\hline 8 & Left \& right panel join & Amina & $\mathrm{O} / \mathrm{L}$ & 0.82 & 76 & 73.55 \\
\hline 9 & Left and right panel join & Morina & $\mathrm{O} / \mathrm{L}$ & 0.79 & 70 & 67.74 \\
\hline 10 & Left and right panel ts \& tc & Mena & $\mathrm{F} / \mathrm{L}$ & 0.53 & 87 & 94.91 \\
\hline 11 & Shoulder panel join & Sharmin & $\mathrm{O} / \mathrm{L}$ & 0.94 & 83 & 115.81 \\
\hline 12 & Shoulder panel ts & Sokina & $\mathrm{F} / \mathrm{L}$ & 0.88 & 89 & 127.14 \\
\hline 13 & Shoulder join & Sahina & $\mathrm{O} / \mathrm{L}$ & 0.92 & 78 & 79.32 \\
\hline 14 & Sleeve panel make & Eima & $\mathrm{O} / \mathrm{L}$ & 0.64 & 64 & 85.33 \\
\hline 15 & Sleeve panel top stitch \& tc & Naher & $\mathrm{F} / \mathrm{L}$ & 0.47 & 103 & 126.12 \\
\hline 16 & Sleeve hem & Momina & $\mathrm{F} / \mathrm{L}$ & 0.42 & 104 & 208.00 \\
\hline 17 & Collar make & Rebeka & $\mathrm{L} / \mathrm{S}$ & 0.79 & 86 & 81.90 \\
\hline 18 & Collar twill tape attach & Shahila & $\mathrm{L} / \mathrm{S}$ & 0.26 & 39 & 106.36 \\
\hline 19 & Collar serving & Lima & $\mathrm{O} / \mathrm{L}$ & 0.43 & 46 & 70.77 \\
\hline 20 & Zipper twill tape make & Orchona & $\mathrm{L} / \mathrm{S}$ & 0.88 & 48 & 68.57 \\
\hline 21 & Zipper twill tape attach & Halima & $\mathrm{L} / \mathrm{S}$ & 0.87 & 57 & 68.40 \\
\hline 22 & Zipper holding tuck & Rojina & $\mathrm{L} / \mathrm{S}$ & 0.66 & 45 & 90.00 \\
\hline 23 & Zipper patch attach at bottom & Bobita & $\mathrm{L} / \mathrm{S}$ & 0.45 & 85 & 89.47 \\
\hline 24 & Collar join & Naher & $\mathrm{L} / \mathrm{S}$ & 1.77 & 118 & 90.77 \\
\hline 25 & Collar join & Shahanara & $\mathrm{L} / \mathrm{S}$ & 1.99 & 127 & 97.69 \\
\hline 26 & Zipper tuck with body & Sabina & $\mathrm{L} / \mathrm{S}$ & 0.36 & 57 & 81.43 \\
\hline 27 & Zipper join with left side & Monzilla & $\mathrm{L} / \mathrm{S}$ & 0.58 & 76 & 91.20 \\
\hline 28 & Zipper join right side & Pervin & $\mathrm{L} / \mathrm{S}$ & 0.76 & 90 & 78.26 \\
\hline 29 & Zipper facing join & Beauty & $\mathrm{L} / \mathrm{S}$ & 0.66 & 80 & 96.00 \\
\hline 30 & Zipper facing o/1 & Hasina & $\mathrm{O} / \mathrm{L}$ & 0.36 & 36 & 72.00 \\
\hline
\end{tabular}

Final Practical output (after sharing of work)

Table 10. Comparison of production before \& after study.

\begin{tabular}{llll}
\hline Parameter & Before Rearrangement & After Rearrangement & After sharing of work \\
\hline No of $\mathrm{m} / \mathrm{c}$ & 30 & 30 & 31 \\
No of manpower & 30 & 30 & 31 \\
Output per hr & 30 & 46 & 68 \\
\hline
\end{tabular}




\section{Results \& Discussion}

Changing from traditional layout to balanced layout model by proper allocation of workers, there are considerable improvements have moved towards us. With final scenario, the best performance results were obtained as summarized in table 10 . The average hourly output of the system increased from 30 to 68 pieces. With reference to scenario, it can be said that the balance of sewing line seems appropriate for all performance measures.

\section{Conclusion}

Skill matrix helps in allocating right person for the right job which helps in achieving desired performance level. It keeps record of all operations an operator had done in the past and efficiency level in each operation. Engineers / line supervisors need minimum time to find and select most efficient operators for an operation from the pull of operators. For line balancing, operators can be selected according to work content. When someone is absent, supervisor can easily find suitable person from the skill matrix table and replace. To analyses the skill availability and distribution throughout the factory. This can be compared with the skill requirement for a particular time period and shortage/excess skill availability to achieve at the training requirement. So productivity can be achieved by allocating skill \& semi-skilled workers to the right place and unskilled operator should be trained properly.

\section{References}

[1] Chen J.C., Chen C.C., Lin Y.J., Lin C.J., and Chen T.Y. Assembly Line Balancing Problem of Sewing Lines in Garment Industry. International Conference on Industrial Engineering and Operations Management, Bali, Indonesia, 2014.

[2] Eryürük S.H., Clothing assembly line design using simulation and heuristic line balancing techniques, Ege University Textile and Apparel Research \& Application Center, 2012.

[3] Eryuruk S. H, Kalaoglu F. and Baskak M. Assembly Line Balancing in a Clothing Company. FIBRES \& TEXTILES in Eastern Europe, 2008.

[4] Jithendrababu B. L., RenjuKurian and Pradeepmon T.G. Balancing Labor Intensive Assembly Line Using Genetic Algorithm. International Journal of Innovative Research in Science, Engineering and Technology, 2013.

[5] Jaganathan V. P. Line balancing using largest candidate rule algorithm in a garment industry: a case study. International Journal of Lean Thinking, 2014.
[6] Chan K.C.C, Hui P.C.L., Yeung K.W., Ng F.S.F. (1998). Handling the assembly line balancing problem in the clothing industry using a genetic algorithm, International Journal of Clothing Science and Technology, Vol.10, pp. 21-37.

[7] Tyler D. J. (1991). Materials Management In Clothing Production, BSP Professional Books Press, London.

[8] Cooklin G. (1991). Introduction to Clothing Manufacturing, Blackwell Science, Oxford, p. 104.

[9] Chuter, A. J. (1988). Introduction to Clothing Production Management, Blackwell Science, 1988.Oxford, pp. 60-63.

[10] Babu V.R. (2011), Industrial engineering in apparel production, Woodhead Publishing Series in Textiles, 129.

[11] Robbins S.P., 1985, "Organizational Behavior- Controversies and Applications" (2nd edition), Prentice-Hall of India (Pvt.) Ltd., New Delhi, 288-292.

[12] Tersine R.J. Production/Operations Management: Concepts, Structure and Analysis, pp.352-374, 1985.

[13] Ramdass K. and Kruger D. The effect of time variations in assembly line balancing: lessons learned in the clothing industry in South Africa. Unisa Institutional Repository, South Africa, 2010.

[14] Bryton, B. Balancing of a Continuous Production Line, M.S. Thesis, Northwestern University, Evanson, ILL. 1954.

[15] Salveson M. E. The Assembly Line Balancing Problem, Journal of Industrial Engineering, 6 (3), pp. 18-25, 1955.

[16] Helgeson W. P., Birnie D. P. Assembly Line Balancing Using the Ranked Positional Weight Technique. Journal of Industrial Engineering, Vol. 12 (6), pp. 384-398, 1961.

[17] Arcus A. L. COMSOAL: A Computer Method of Sequencing for Assembly Lines. International Journal of Production Research, 4 (4), pp. 259-277, 1966.

[18] Bartholdi J.J. Balancing two-sided assembly lines: A case study. International Journal of Production Research, Vol.31, 10, pp.2447-2461, 1993.

[19] Liu C.M., Chen C.H. Multi-section electronic assembly line balancing problems: A case study. International Journal of Product Planning \& Control. 13 451-461, 2002.

[20] Yolmeh Abdolmajid and Kianfar Farhad. An efficient hybrid genetic algorithm to solve assembly line balancing problem with sequence dependent setup times. International Journal of Computers \& Industrial Engineering, Elsevier-Volume 62, Issue 4, Pages 839- 1144, May 2012.

[21] Narkhedkar R. N., Vishnu Dhorugade and Sonavane M.J. Skill matrix: Effective tool to boost productivity. Indian textile journal, 2011. 